\title{
RESEÑAS DE EVENTOS
}




\section{COLOQUIO DE HISTORIA DE LA EDUCACIÓN COLOMBIANA \\ Tunja-Boyacá. 12 al 15 de agosto de 2008}

Este evento se desarrolló entre el 12 y 15 de agosto de 2008 en el marco del XIV Congreso Colombiano de Historia, efectuado en la Universidad pedagógica y Tecnológica de Colombia. En el "VII Coloquio de Historia de la Educación Colombiana" se presentaron 21 ponencias que aparecen registradas, con sus respectivos resúmenes y textos completos en las memorias publicadas en CD: "Colección Memorias de Historia".

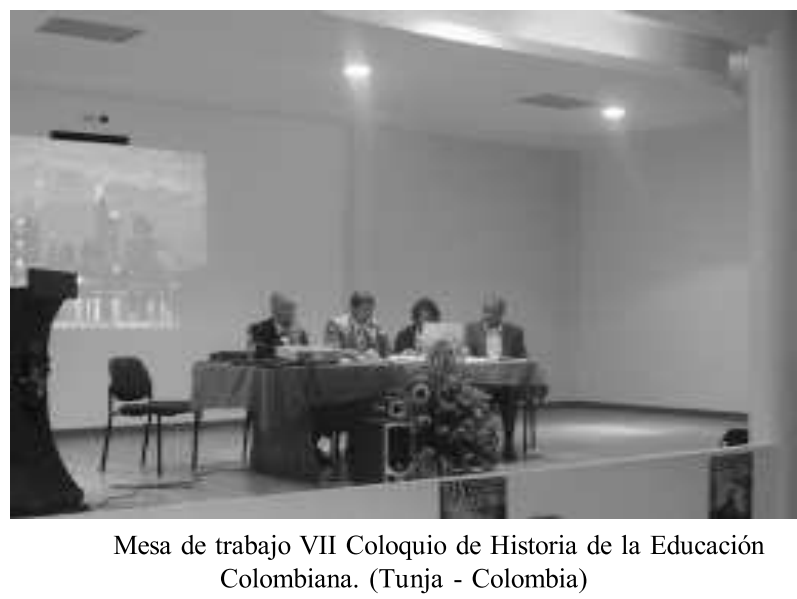

Como temática central este coloquio se orientó hacia la construcción de la nación. Así, desde diversas perspectivas se pretendió mirar cómo lo institucional, las políticas públicas, las prácticas docentes, la formación de maestros y el surgimiento de ciertos saberes incidían en la construcción de un ciudadano para responder a la sociedad del momento.

Algunas de las tendencias que se pudieron apreciar en este evento fueron: formación de maestros, educación superior, educación y ciudadanía en la construcción de nación, historia de personajes a través de los cuales se analiza un contexto, Iglesia y educación, la educación para los excluidos, la independencia y el sistema educativo. Uno de los temas innovadores fue la historia de la educación para la infancia, el que se mira desde la normatividad que según los autores es el inicio del reconocimiento del Estado por este sector social y por establecer políticas para la formación de los niños. Así mismo el concepto de nación y ciudadanía fue planteado por varios ponentes, para quienes la educación se convirtió en un medio significativo para la formación de ciudadano en la perspectiva de construcción de la nación. Otros trabajos hacen alusión al surgimiento del sistema Universitario en Colombia lo que es significativo para comprender la emergencia de políticas universitarias que dan las bases para su desarrollo en la segunda mitad del siglo veinte e incluso hasta nuestros días. Deben resaltarse también las temáticas que abordan la historia de instituciones, a través de las cuales se pretende ver un contexto social y político.

Otras temáticas en las que centran su atención los ponentes es en las relaciones iglesia-estado y su papel educativo; así mismo la historia de los saberes que es fundamental para dar respuesta a la formación física como parte del bienestar del individuo. Por otra parte, temas como la formación de maestros, la autonomía universitaria, el cómo las logias liberales tenían una particularidad para su formación son importantes para comprender la dinámica de la sociedad en el tiempo. Este y otros 
eventos sobre Historia de la educación colombiana ayudará a comprender diversos escenarios de la sociedad que no han sido abordados y que son importantes para comprender las experiencias de los disímiles grupos sociales y el cómo la sociedad puede proyectar diversidad de futuros posibles.

Olga Yanet Acuña Rodríguez Universidad Pedagógica y Tecnológica de Colombia Grupo de Investigación HISULA-SHELA

\section{SIMPOSIO "TEXTOS Y MANUALES UNIVERSITARIOS" REALIZADO EN EL CONGRESO "TEXTOS, AUTORES Y BIBLIOTECAS"}

Argentina, Universidad Nacional de Córdoba. 24 al 26 de septiembre de 2008

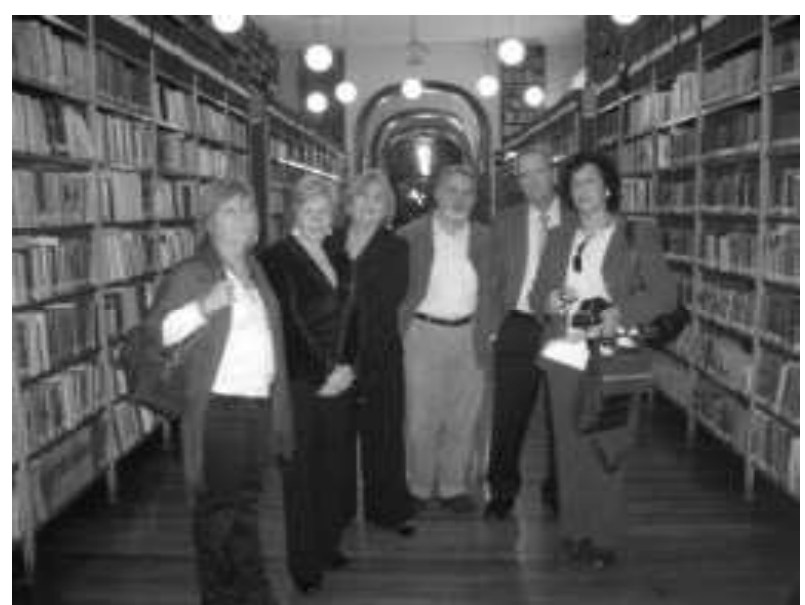

Grupo en la Biblioteca Mayor de Córdoba - Argentina. 2008

El Simposio "Textos y manuales universitarios" estuvo coordinado por la Dra. María Cristina Vera de Flachs y Dra. Diana Soto Arango. Se presentaron 17 trabajos de investigadores procedentes de seis países: Argentina, Brasil, Colombia, España, México y Uruguay.

Los textos presentados correspondieron a los periodos del Siglo XVIII, XIX, XX y XXI. Destacamos que las grandes temáticas correspondieron a los textos del período colonial; la evolución en la enseñanza; bibliotecas del siglo XIX; los tratados y manuales en el campo de la medicina, arquitectura, Historia de la educación, geografía que fueron analizados desde diferentes perspectivas metodológicas. Además, se analizó el texto escolar en la educación básica primaria y secundaria dentro de las nuevas metodologías y tecnologías que llevan a replantear el concepto del texto escolar por el de texto didáctico. Se destaca por su entidad la nueva metodología que se planteo para el análisis de los textos escolares.

Diana Elvira Soto Arango

Universidad Pedagógica y Tecnológica de Colombia Directora Grupo de Investigación HISULA-SHELA 


\section{CONGRESO DE HISTORIA}

Mentalidades, Representaciones e Imaginarios

Hacia el Bicentenario de la Independencia (1810-2010).

Hacia el Cuatricentenario del Santo Cristo de la Grita (1610-2010).

En el 190 Aniversario de la Campaña Libertadora (1819-2009)

San Cristóbal-Estado Táchira-Venezuela. 16 al 19 de abril de 2009

Atendiendo la invitación de la Universidad de Los Andes-Táchira, del Grupo de investigación de Historia de la Educación y Representaciones (HEDURE), de la Revista Heurística, (revista digital de Historia de la Educación y de la Sociedad Bolivariana del Táchira, una delegación de docentes yestudiantes (16 personas) del Doctorado en Historia de la Educación, del Doctorado en Historia y de la Maestría en Historia de la UPTC, y de la Academia Boyacense de Historia, asistieron como ponentes y participantes al PRIMER CONGRESO DE HISTORIA que se realizó en la Ciudad de San Cristóbal

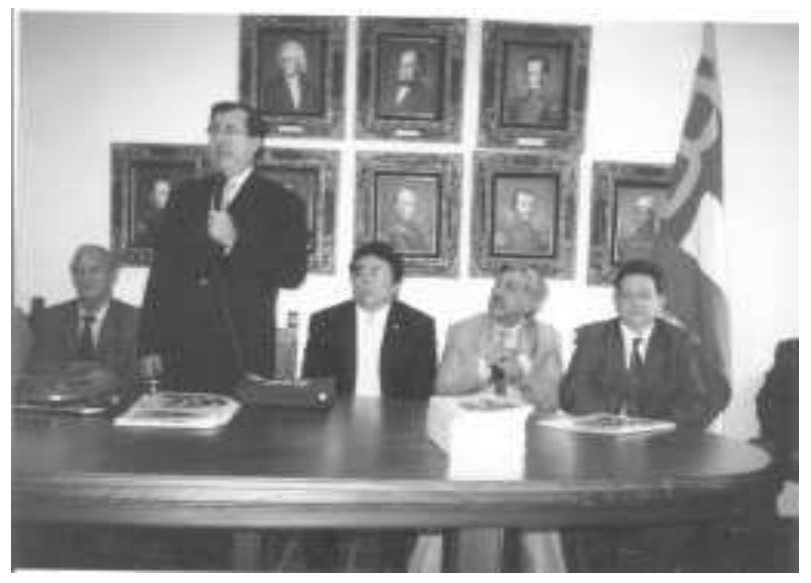

Mesa de trabajo I Congreso de Historia (Táchira -Venezuela Colombia) Venezuela y que trazó como misión el iniciar la conmemoración del año jubilar del Bicentenario de la Independencia, del Cuatricentenario del Santo Cristo de la Grita y del 190 Aniversario de La Campaña Libertadora. Fueron Objetivos del Congreso: analizar las teorías y métodos de la Ciencia de la Historia, teorías y tendencias actuales, el estado del arte de las tendencias historiográficas y fomentar las investigaciones históricas, Redes y Comunidades discursivas de la Ciencia de la Historia en Venezuela y en América Latina.

El Congreso contó con la Dirección General del Dr. Reinaldo Rojas (Presidente Honorario-Vice Ministro de la Educación Superior de Venezuela) y la Coordinación del Doctor José Pascual Mora García (Presidente de la Academia de Historia del Táchira y de la Sociedad Bolivariana del Táchira), y de los Miembros integrantes de la Comisión Científica Nacional, la Comisión Científica Internacional y del Comité Ejecutivo. Con la asistencia de 270 personas y la defensa de 173 ponencias los representantes de 12 Universidades en el área de las Ciencias Históricas de Colombia, Francia, España, México, Brasil y el anfitrión Venezuela. Cinco Mesas de Trabajo: No.1. Historiografía de la Independencia. Estado del arte sobre el proceso independentista y temas afines; No.2. La Historia de las mentalidades. Representaciones colectivas e imaginarios sociales: el "utillaje mental" en la independencia, la invención de la tradición y la construcción del héroe nacional; No.3. Las narrativas histórico-literarias y de ficción sobre la independencia: La historia de la dialéctica de la negación, enfoques de género y afro descendientes; No.4.Historia de la Ciencia, la técnica y la tecnología y No.5. Historia Regional y local. Cronistas y narradores orales. 
Fueron Ponentes los estudiantes de la UPTC del Doctorado en Historia de la Educación RUDECOLOMBIA, el Padre José Herson Mejía Ramírez "la Encíclica populorum Progressio y su preocupación por la justicia social de S.S. Pablo VI y el Profesor Jorge Enrique Duarte Acero "De la Virgen del Rosario de Chiquinquirá en Colombia a la Virgen del Rosario de la Chiquinquirá de Aregue en Venezuela 15861870" y de la Maestría en Historia.

El Doctor Olmedo Vargas Hernández, Director del Doctorado en Historia, dictó la Conferencia inaugural "Historia de las Ideas en Boyacá: mentalidad e historiografía renacentista en las letras Boyacenses" (jueves 16, en reemplazo del Dr. Javier Ocampo López quien no pudo asistir al evento). Y la conferencia Magistral Central en la Sesión Solemne de la Academia de Historia del Táchira para conmemorar el 19 de abril de 1810la independencia de Venezuela la dicto, el Doctor Jorge Enrique Duarte Acero, sobre "La Campaña Libertadora de Boyacá 1817-1819 .La batalla del Pantano de Vargas, (el 25 de julio de 1819) y el triunfo del Coronel Patriota Venezolano Juan José Rondón”. En ese mismo evento se hizo imposición de la Magna Orden de la Sociedad Bolivariana del Táchira al Dr. Olmedo Vargas Hernández, en calidad de Ex Rector de UPTC, por la firma en el 2001 del Convenio de Cooperación Internacional UPTC-GOBIERNO de la República Bolivariana De Venezuela (Universidad Pedagógica Experimental Libertador UPEL Barquisimeto Estado Lara), hoy vigente y que ha permitido la realización de pasantias de estudiantes del Doctorado.

El domingo 19 de abril, día de la Independencia de la República Bolivariana de Venezuela, el Dr. Cesar Pérez Vivas, Gobernador del Estado Táchira hizo lectura del pergamino con el Decreto (Gaceta Departamental) que entrega la Casa Sede de la Sociedad Bolivariana del Táchira y de la Academia de Historia del Táchira y la declara Patrimonio Histórico y Cultural del Táchira y en el salón principal de eventos se devela la placa en mármol que dedica su nombre al Dr. Javier Ocampo López, docente de la UPTC como "el Historiador y académico de las dos Repúblicas Hermanas : Colombia y Bolivariana de Venezuela. En el Congreso de Historia se destaco la participación de los Cronistas ynarradores orales en calidad de ponentes, entre ellos al Dr. J.J. Villamizar Molina, Decano de los Cronistas de Venezuela y de la presencia de la académica Venezolana Dra. Edda O. Samudio Miembro de la Asociación de Historiadores Latinoamericanistas Europeos y Miembro Correspondiente de la Academia Colombiana de Historia, con la ponencia "Proceso histórico de la Institucionalización de los Estudios Superiores en Mérida-Siglo XVIII.

Una de las principales conclusiones del evento apunto a seguir reflexionando desde las Academias e Instituciones educativas y Universidades acerca de la historiografía de la Independencia (Bicentenario 1810 -2010), del proceso independentista Neogranadino (Campaña libertadora 1819-2019) y de las mentalidades, representaciones colectivas e imaginarios sociales, como elemento historiográfico para la recuperación de la memoria histórica en el contexto Bolivariano y latinoamericano.

Jorge Enrique Duarte Acero Doctorando en Historia de la EducaciónRUDECOLOMBIA 
HISTORIA DE LOS CONGRESOS DE SHELA

Argentina, Brasil, Colombia, España, México, Perú, Venezuela, 28 de abril del 2009

Estos congresos activos desde 1992, inauguran su ciclo académico, con el " $I$ Congreso Iberoamericano de Historiadores de la Educación Latinoamericana", que se realizó en Bogotá, Colombia, del 02 al 05 de septiembre de 1992, bajo la coordinación de Diana Soto Arango, organizadora de la Red de Historiadores de la Educación Latinoamericana, lo cual constituye uno de los primeros eventos y acciones de la "prehistoria" de la fundación de la Sociedad de Historia de la Educación de Latinoamerica na (SHELA). Este primer congreso tenía su antecedente en el "I

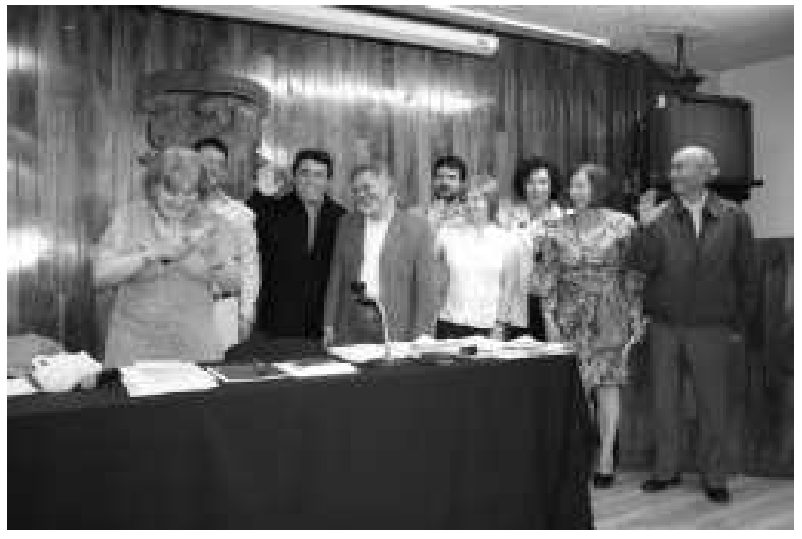

Junta Directiva de la Sociedad de Historia de la Educación Latinoamericana (2009). Coloquio de Historia de la Educación en Colombia”, organizado por Diana Soto Arango, en Bogotá en el año 1989.

En 1994 fue fundada la Sociedad de Historia de la Educación Latinoamericana SHELA, durante la celebración del "II Congreso Iberoamericano de Historiadores de la Educación Latinoamericana", realizado en UNICAMP, en la ciudad de Campinas, Brasil, del 11 al 15 de septiembre (de 1994), bajo la coordinación de Silvio Sánchez Gamboa. Se eligió, por la asamblea de los socios fundadores, como presidenta a la Dra. Diana Soto Arango (Colombia); agenciando su representación desde su país de origen. La Sociedad obtuvo el Registro Legal Número 145-250 de junio de 1995. SHELA, constituida como sociedad, bajo la orientación académica del grupo base que organizó el I Congreso Iberoamericano, ha sido responsable de la organización de los siguientes congresos: "III Congreso Iberoamericano de Historia de la Educación Latinoamericana", realizado en la Universidad Central de Venezuela y la Universidad Pedagógica Experimental Libertador, en Caracas, Venezuela del 9 al 15 de junio de 1996, bajo la coordinación de Magaldy Téllez. "IV Congreso Iberoamericano de la Educación Latinoamericana", celebrado en la Universidad Católica de Santiago de Chile, del 25 al 29 de mayo de 1998, con la coordinación de Dr. Luis Celis (Chile) quien fuera elegido como presidente de SHELA.

El "V Congreso Iberoamericano de Historia de la Educación Latinoamericana", se realizó en la Universidad Nacional de Piura, en Piura, Perú, del 25 al 29 de noviembre de 2002, bajo la coordinación de la académica Ruth Santibáñez de esa Universidad. En este evento se eligió la mesa directiva que encabezó la Dra. María Cristina Vera de Flachs (Argentina) quien asumió la presidencia. El "VI Congreso Iberoamericano de Historia de la Educación Latinoamericana", realizado en la Universidad de Guadalajara, en 
Guadalajara, México, del 29 al 31 de octubre de 2007, bajo la coordinación del Dr. Armando Martínez Moya. En este evento en asamblea estatutaria se elige presidente de la Sociedad al Dr. José Rubens Jardilino (Brasil).

Es importante dilucidar que dentro de la historiografía educativa latinoamericana que SHELA ha venido promoviendo, dialogando y difundiendo, el campo analítico por excelencia que se ha trabajo es el de la educación superior, en particular la historia de las universidades hispanoamericanas, así como los colegios mayores coloniales, a partir de los diferentes referentes y dimensiones de su análisis.

También debemos anotar que en el tercer y cuarto congresos surgió una tendencia de compañeros de la sociedad representada por parte de algunos miembros de las delegaciones de Argentina y de Venezuela, de organizar unos Congresos más amplios, buscando privilegiar la cobertura. Otra tendencia, consideró que los espacios de discusión debían de darse de manera más específica y cuidando la discusión y calidad de los eventos. Esta fue la posición mayoritaria (95\%), recomendando además la creación de sociedades nacionales, que estaba impulsando SHELA. En el caso de la otra posición se consideró lógico que se conformara otra Sociedad con ese carácter amplio que esa tendencia de compañeros proponía, pero que SHELA seguiría con la modalidad de inscripción y representación individual cuidando el vínculo entre participación y proyecto de investigación. La nueva sociedad se creó en el Congreso que realizaron en Costa Rica en el año 2000.

Por lo tanto, consideramos que la actuación de ambas sociedades contribuyen al desarrollo de la investigación en la Historia de la Educación Latinoamericana. En nuestro caso, debemos buscar, al cumplir SHELA en el 2009, 15 años de existencia y 18 años de celebración de eventos hispanoamericanos e internacionales, consolidar y proyectar nuestro esfuerzo académico.

En 2009 SHELA organizará la séptima edición internacional de sus congresos estatutarios, los cuales le han permitido nuclear y consolidar un importante y representativo grupo de investigadores que han contribuido a darle forma y resultados importantes, pues se han involucrado en organización regional y continental, en la promoción de la investigación, en responsabilidades directivas y académicas y en la publicación y discusión de diversas temáticas historiográficas.

El congreso próximo, se realizará en Sao Paulo, Brasil, del 23 al 25 de agosto, bajo la coordinación de Dr. José Rubens Jardilino, con el auspicio de las siguientes instituciones de Educación Superior: Universidad Nove de Julho, Universidad Presbiteriana Mackenzie, Universidad Ciudad de Sao Paulo, Universidad Sao Caetano del Sur en Brasil; las universidades de León, Valencia, Sevilla y Pablo de Olavide en España; Universidades de Guadalajara y Tamaulipas en México; Universidad de Trujillo en Perú; Universidad Nacional Experimental Libertador- Instituto Pedagógico Barquisimeto, Universidad de Los Andes-Táchira en Venezuela; Universidad Pedagógica de Honduras, la Red de Universidades Públicas de Colombia RUDECOLOMBIA, con 80 grupos de investigación avalados por COLCIENCIAS para Iberoamérica. 
La actual junta directiva está conformada por: Dr. José Rubens Jardilino (Presidente, Brasil); Dr. José Pascual Mora (Vice-presidente, Venezuela); Dr. Armando Martínez Moya (Secretario, México); Dr. Carlos Valencia (Tesorero, Colombia); Dr. Elmer Robles Ortiz (Fiscal, Perú), Dra. Remedios Ferrero Micó (Vocal, España); Dra. María Isabel Lafuente (Vocal, España); Dra. María Cristina Vera (Vocal, Argentina), Dra. Diana Soto Arango (Vocal, Colombia).

Finalmente, indicamos que la Sociedad de Historia de la Educación Latinoamericana, SHELA, ha tenido como Misión posicionar en el contexto euroamericano e internacional el desarrollo investigativo de cada uno de sus integrantes, consolidando una comunidad académica e investigativa, caracterizada por su compromiso en la formación de historiadores de la educación en Latinoamérica. Esta formación se impulsa desde la producción de conocimiento en la racionalidad científica, coherencia e identidad regional, contribuyendo a valorar las ideas científicas, filosóficas, políticas y pedagógicas con los principios, fines y objetivos que surgen a partir de los estudios de la historia de la educación latinoamericana.

Creemos que un rasgo que nos distingue y visibiliza en la comunidad académica euroaméricana, es la conformación de los grupos de investigación, objeto central de la creación de Sociedad de Historia de la Educación Latinoamericana. Esta situación ha posibilitado una importante política de difusión a través de publicaciones como la Revista de Historia de la Educación Latinoamericana, publicación con arbitraje internacional y el Boletín que la sociedad por lo regular edita previamente a los eventos en los cuales participa. SHELA organiza simposios, congresos y otros eventos en diferentes lugares del mundo. Entre otros podemos destacar: en Liverpool (Inglaterra); en Moscú (Rusia); Macau (China); Bratislava (Eslovaquia); Tamaulipas, Guadalajara (México); Córdoba (Argentina); Varsovia (Polonia); Sevilla (España); Cartagena (Colombia). También, se publican regularmente colecciones de libros, cuyos autores son principalmente miembros de SHELA, en colaboración con otros importantes historiadores hispanoamericanos.

\author{
Junta Directiva de la Sociedad de Historia de la Educación Latinoamericana \\ Argentina, Brasil, Colombia, España, México, Perú, Venezuela, \\ 28 de abril del 2009 \\ www.shela-edu.org www.rhela.rudecolombia.edu.co
}

